\title{
Uniform Treatment of Jensen's Inequality by Montgomery Identity
}

\author{
Tahir Rasheed, ${ }^{1}$ Saad Ihsan Butt $\left(\mathbb{D},{ }^{1}\right.$ Đilda Pečarić, ${ }^{2}$ Josip Pečarić (D), ${ }^{3}$ \\ and Ahmet Ocak Akdemir ${ }^{4}{ }^{4}$ \\ ${ }^{1}$ COMSATS University Islamabad, Lahore Campus, Lahore, Pakistan \\ ${ }^{2}$ Department of Media and Communication, University North, Trg dr. Žarka Dolinara 1, Koprivnica, Croatia \\ ${ }^{3}$ Peoples Friendship University of Russia (RUDN University), 6 Miklukho-Maklaya St, Moscow 117198, Russia \\ ${ }^{4}$ Department of Mathematics, Faculty of Arts and Sciences, A ğr İbrahim Çeçen University, A ̆grl, Turkey
}

Correspondence should be addressed to Ahmet Ocak Akdemir; aocakakdemir@gmail.com

Received 26 February 2021; Revised 2 April 2021; Accepted 12 April 2021; Published 17 May 2021

Academic Editor: Xiaolong Qin

Copyright ( 12021 Tahir Rasheed et al. This is an open access article distributed under the Creative Commons Attribution License, which permits unrestricted use, distribution, and reproduction in any medium, provided the original work is properly cited.

We generalize Jensen's integral inequality for real Stieltjes measure by using Montgomery identity under the effect of $\mathfrak{n}-$ convex functions; also, we give different versions of Jensen's discrete inequality along with its converses for real weights. As an application, we give generalized variants of Hermite-Hadamard inequality. Montgomery identity has a great importance as many inequalities can be obtained from Montgomery identity in $q$-calculus and fractional integrals. Also, we give applications in information theory for our obtained results, especially for Zipf and Hybrid Zipf-Mandelbrot entropies.

\section{Introduction}

Convex functions have a great importance in mathematical inequalities, and the well-known Jensen's inequality is the characterization of convex functions. Jensen's inequality for differentiable convex functions plays a significant role in the field of inequalities as several other inequalities can be seen as special cases of it. One can find the application of Jensen's discrete inequality in discrete-time delay systems in [1].

Taking into consideration the tremendous applications of Jensen's inequality in various fields of mathematics and other applied sciences, the generalizations and improvements of Jensen's inequality have been a topic of supreme interest for the researchers during the last few decades as evident from a large number of publications on the topic (see [2-4] and the references therein).

The well-known Jensen's inequality asserts that for the function $\Gamma$ it holds that

$$
\Psi\left(\frac{1}{P_{\mathfrak{m}}} \sum_{\mathcal{F}=1}^{\mathfrak{m}} p_{\mathcal{F}} x_{\mathcal{F}}\right) \leq \frac{1}{P_{\mathfrak{m}}} \sum_{\mathcal{F}=1}^{\mathfrak{m}} p_{\mathcal{f}} \Psi\left(x_{\mathcal{f}}\right),
$$

if $\Psi$ is a convex function on interval $I \subset \mathbb{R}$, where $p_{g}$ are positive real numbers and $x_{\mathcal{f}} \in I(\mathscr{F}=1, \ldots, \mathfrak{m})$, while $P_{\mathfrak{m}}=\sum_{q=1}^{\mathfrak{m}} p_{q}$.

However, the well-known integral analogue of Jensen's inequality is as follows.

Theorem 1. Let $\hbar:[a, b] \longrightarrow[\alpha, \beta]$ be a continuous function and $\lambda:[a, b] \longrightarrow \mathbb{R}$ be an increasing and bounded function with $\lambda(a) \neq \lambda(b)$. Then, for every continuous convex function $\Psi:[\alpha, \beta] \longrightarrow \mathbb{R}$, the following inequality holds:

$$
\Psi(\widetilde{\hbar}) \leq \frac{\int_{a}^{b} \Psi(\hbar(\zeta)) \mathrm{d} \lambda(\zeta)}{\int_{a}^{b} \mathrm{~d} \lambda(\zeta)},
$$

where

$$
\widetilde{\hbar}=\frac{\int_{a}^{b} \hbar(\zeta) \mathrm{d} \lambda(\zeta)}{\int_{a}^{b} \mathrm{~d} \lambda(\zeta)} \in[\alpha, \beta] .
$$

There are several inequalities coming from Jensen's inequality both in integral and discrete cases which can be 
obtained by varying conditions on the function $\hbar$ and measure $\lambda$ defined in Theorem 1 .

Montgomery identity is used in quantum calculus or $q$-calculus. There are different identities of Montgomery, and several inequalities of Ostrowski type were formulated by using these identities. Budak and Sarikaya established the generalized Montgomery-type identities for differential mappings in [5]. Applications of Montgomery identity can be found in fractional integrals as well as in quantum integral operators. Here we utilize Montgomery's identity for the generalization of Jensen's inequality. In [6], Cerone and Dragomir developed a systematic study which produced some novel inequalities. Several interesting results related to inequalities and different types of convexity can be found in [7-21]. The class of convex functions is a very useful concept that has become a focus of interest for researchers in statistics, convex programming, and many other applied disciplines, as well as in inequality theory. The readers can find some motivated findings related to convex functions and some new integral inequalities in [22-27].

In [28], Khan et al. have mentioned about $\mathfrak{n}$-convex functions as follows.

Definition 1. A function $f: I \longrightarrow \mathbb{R}$ is called convex of order $\mathfrak{n}$ or $\mathfrak{n}$-convex if for all choices of $(n+1)$ distinct points $x_{i}, \ldots, x_{i+n}$ we have $\Delta_{(n)} f\left(x_{i}\right) \geq 0$.

If $n$-th order derivative $f^{(n)}$ exists, then $f$ is $\mathfrak{n}$-convex if and only if $f^{(n)} \geq 0$. For $1 \leq k \leq(n-2)$, a function $f$ is $\mathfrak{n}$-convex if and only if $f^{(k)}$ exists and is $(n-k)$-convex.

In the present paper, we will use Montgomery identity that is presented as following.

Theorem 2. Let $\mathfrak{n} \in \mathbb{N}, \Psi: I \longrightarrow \mathbb{R}$ be such that $\Psi^{(\mathfrak{n}-1)}$ is absolutely continuous, $I \subset \mathbb{R}$ is an open interval, and $\alpha, \beta \in I$, $\alpha<\beta$. Then, the following identity holds:

$$
\Psi(x)=\frac{1}{\beta-\alpha} \int_{\alpha}^{\beta} \Psi(t) \mathrm{d} t+\sum_{\ell=0}^{\mathfrak{n}-2} \frac{\Psi^{(\ell+1)}(\alpha)}{\ell !(\ell+2)} \frac{(x-\alpha)^{\ell+2}}{\beta-\alpha}-\sum_{\ell=0}^{\mathfrak{n}-2} \frac{\Psi^{(\ell+1)}(\beta)}{\ell !(\ell+2)} \frac{(x-\beta)^{\ell+2}}{\beta-\alpha}+\frac{1}{(\mathfrak{n}-1) !} \int_{\alpha}^{\beta} R_{\mathfrak{n}}(x, s) \Psi^{(\mathfrak{n})}(s) \mathrm{d} s,
$$

where

$$
R_{\mathfrak{n}}(x, s)= \begin{cases}-\frac{(x-s)^{\mathfrak{n}}}{\mathfrak{n}(\beta-\alpha)}+\frac{x-\alpha}{\beta-\alpha}(x-s)^{\mathfrak{n}-1}, & \alpha \leq s \leq x, \\ -\frac{(x-s)^{\mathfrak{n}}}{\mathfrak{n}(\beta-\alpha)}+\frac{x-\beta}{\beta-\alpha}(x-s)^{\mathfrak{n}-1}, & x<s \leq \beta .\end{cases}
$$

\section{Generalization of Jensen's Integral Inequality by Using Montgomery Identity}

Before giving our main results, we consider the following assumptions that we use throughout our paper:

$A_{1}$ Let $\hbar:[a, b] \longrightarrow \mathbb{R}$ be continuous function.

$A_{2}$ Let $\lambda:[a, b] \longrightarrow \mathbb{R}$ be a continuous function or the functions of bounded variation such that $\lambda(a) \neq \lambda(b)$.
2.1. New Generalization of Jensen's Integral Inequality. In our first main result, we employ Montgomery identity to obtain the following real Stieltjes measure's theoretical representations of Jensen's inequality.

Theorem 3. Let $g, \lambda$ be as defined in $A_{1}, A_{2}$ such that $\hbar([a, b]) \subset[\alpha, \beta]$. Also, let $\Psi:[\alpha, \beta] \longrightarrow \mathbb{R}$ be such that for $\mathfrak{n} \geq 1, \Psi^{(\mathfrak{n}-1)}$ is absolutely continuous. If $\Psi$ is $\mathfrak{n}$-convex such that

$$
R_{\mathfrak{n}}(\widetilde{\hbar}, s) \leq \frac{\int_{a}^{b} R_{\mathfrak{n}}(\hbar(\zeta), s) \mathrm{d} \lambda(\zeta)}{\int_{a}^{b} \mathrm{~d} \lambda(\zeta)}, \quad s \in[\alpha, \beta]
$$

with $\tilde{\hbar}$ and $R_{\mathfrak{n}}(x, s)$ as defined in (3) and (5), respectively, then we have

$$
\begin{aligned}
& \Psi(\widetilde{\hbar})-\frac{\int_{a}^{b} \Psi(\hbar(\zeta)) \mathrm{d} \lambda(\zeta)}{\int_{a}^{b} \mathrm{~d} \lambda(\zeta)} \leq \sum_{\ell=0}^{\mathfrak{n}-2} \frac{1}{\ell !(\ell+2)(\beta-\alpha)} \times \\
& {\left[\Psi^{(\ell+1)}(\alpha)\left((\widetilde{\hbar}-\alpha)^{(\ell+2)}-\frac{\int_{a}^{b}(\hbar(\zeta)-\alpha)^{(\ell+2)} \mathrm{d} \lambda(\zeta)}{\int_{a}^{b} \mathrm{~d} \lambda(\zeta)}\right)-\Psi^{(\ell+1)}(\beta)\left((\widetilde{\hbar}-\beta)^{(\ell+2)}+\frac{\int_{a}^{b}(\hbar(\zeta)-\beta)^{(\ell+2)} \mathrm{d} \lambda(\zeta)}{\int_{a}^{b} \mathrm{~d} \lambda(\zeta)}\right)\right] .}
\end{aligned}
$$


Proof. As $\Psi^{(\mathfrak{n}-1)}$ is absolutely continuous for $(\mathfrak{n} \geq 1)$, we can use the representation of $\Psi$ using Montgomery identity (4) and can calculate

$$
\Psi(\tilde{\hbar})=\frac{1}{\beta-\alpha} \int_{\alpha}^{\beta} \Psi(\zeta) \mathrm{d}(\zeta)+\sum_{\ell=0}^{\mathfrak{n}-2} \frac{\Psi^{(\ell+1)}(\alpha)}{\ell !(\ell+2)} \frac{(\tilde{\hbar}-\alpha)^{\ell+2}}{\beta-\alpha}-\sum_{\ell=0}^{\mathfrak{n}-2} \frac{\Psi^{(\ell+1)}(\beta)}{\ell !(\ell+2)} \frac{(\widetilde{\hbar}-\beta)^{\ell+2}}{\beta-\alpha}+\frac{1}{(\mathfrak{n}-1) !} \int_{\alpha}^{\beta} R_{\mathfrak{n}}(\tilde{\hbar}, s) \Psi^{(\mathfrak{n})}(s) \mathrm{d} s
$$

The integration of the composition of functions $\Psi \circ \hbar$ for the real measure $\lambda$ on $[a, b]$ gives

$$
\begin{aligned}
\frac{\int_{a}^{b} \Psi(\hbar(\zeta)) \mathrm{d} \lambda(\zeta)}{\int_{a}^{b} \mathrm{~d} \lambda(\zeta)}= & \frac{1}{\beta-\alpha} \int_{\alpha}^{\beta} \Psi(\zeta) \mathrm{d}(\zeta)+\sum_{\ell=0}^{\mathfrak{n}-2} \frac{\Psi^{(\ell+1)}(\alpha)}{\ell !(\ell+2)} \frac{\int_{a}^{b}(\hbar(\zeta)-\alpha)^{(\ell+2)} \mathrm{d} \lambda(\zeta)}{(\beta-\alpha) \int_{a}^{b} \mathrm{~d} \lambda(\zeta)} \\
& -\sum_{\ell=0}^{\mathfrak{n}-2} \frac{\Psi^{(\ell+1)}(\beta)}{\ell !(\ell+2)} \frac{\int_{a}^{b}(\hbar(\zeta)-\beta)^{(\ell+2)} \mathrm{d} \lambda(\zeta)}{(\beta-\alpha) \int_{a}^{b} \mathrm{~d} \lambda(\zeta)}+\frac{1}{(\mathfrak{n}-1) !} \int_{\alpha}^{\beta} R_{\mathfrak{n}}(\hbar(\zeta), s) \Psi^{(\mathfrak{n})}(s) \mathrm{d} s .
\end{aligned}
$$

Now computing the difference $\Psi(\widetilde{\hbar})-\int_{a}^{b} \Psi(\hbar(\zeta))$ $\mathrm{d} \lambda(\zeta) / \int_{a}^{b} \mathrm{~d} \lambda(\zeta)$, we get the following generalized identity involving real Stieltjes measure:

$$
\begin{aligned}
\Psi(\widetilde{\hbar})-\frac{\int_{a}^{b} \Psi(\hbar(\zeta)) \mathrm{d} \lambda(\zeta)}{\int_{a}^{b} \mathrm{~d} \lambda(\zeta)}= & \sum_{\ell=0}^{\mathfrak{n}-2} \frac{1}{\ell !(\ell+2)(\beta-\alpha)} \\
& \times\left[\Psi^{(\ell+1)}(\alpha)\left((\widetilde{\hbar}-\alpha)^{(\ell+2)}-\frac{\int_{a}^{b}(\hbar(\zeta)-\alpha)^{(\ell+2)} \mathrm{d} \lambda(\zeta)}{\int_{a}^{b} \mathrm{~d} \lambda(\zeta)}\right)-\Psi^{(\ell+1)}(\beta)\left((\widetilde{\hbar}-\beta)^{(\ell+2)}+\frac{\int_{a}^{b}(\hbar(\zeta)-\beta)^{(\ell+2)} \mathrm{d} \lambda(\zeta)}{\int_{a}^{b} \mathrm{~d} \lambda(\zeta)}\right)\right] \\
& +\frac{1}{(\mathfrak{n}-1) !} \int_{\alpha}^{\beta}\left(R_{\mathfrak{n}}(\widetilde{\hbar}, s)-\frac{\int_{a}^{b} R_{\mathfrak{n}}(\hbar(\zeta), s) \mathrm{d} \lambda(\zeta)}{\int_{a}^{b} \mathrm{~d} \lambda(\zeta)}\right) \Psi^{(\mathfrak{n})}(s) \mathrm{d} s .(\text { GI. } 1) .
\end{aligned}
$$

Finally, by our assumption, $\Psi^{(\mathfrak{n}-1)}$ is absolutely continuous on $[\alpha, \beta]$; as a result, $\Psi^{(\mathfrak{n})}$ exists almost everywhere. Moreover, $\Psi$ is supposed to be $\mathfrak{n}$-convex, so we have $\Psi^{(\mathfrak{n})}(x) \geq 0$ almost everywhere on $[\alpha, \beta]$. Therefore, by taking into account the last term in generalized identity (GI.1) and integral analogue of Jensen's inequality that is given in (6), we get (7).

In the later part of this section, we will vary our conditions on functions $g$ and Stieltjes measure $\mathrm{d} \lambda$ to obtain generalized variants of Jensen-Steffensen, Jensen-Boas, Jensen-Brunk, and Jensen-type inequalities. We start with the following generalization of Jensen-Steffensen inequality for $n$-convex functions.

Theorem 4. Let $\Psi$ defined in Theorem 3 be $\mathfrak{n}$-convex and $\hbar$ defined in $M_{1}$ be monotonic. Then, the following results hold.

(i) If $\lambda$ defined in $M_{2}$ satisfies

$\lambda(a) \leq \lambda(x) \leq \lambda(b), \quad \forall x \in[a, b], \lambda(b)>\lambda(a)$,

then for even $\mathfrak{n} \geq 3$, (6) is valid.

(ii) Moreover, if (6) is valid and the function

$$
H(x):=\sum_{\ell=0}^{\mathfrak{n}-2}\left(\frac{1}{\ell !(\ell+2)(\beta-\alpha)}\right)\left(\Psi^{(\ell+1)}(\alpha)(x-\alpha)^{\ell+2}-\Psi^{(\ell+1)}(\beta)(x-\beta)^{\ell+2}\right)
$$


is convex, then we get inequality (2) which is called generalized Jensen-Steffensen inequality for $\mathfrak{n}$-convex function.

Proof. (i) By applying second derivative test, we can show that the function

$R_{\mathfrak{n}}(x, s)$ is convex for even $\mathfrak{n}>3$. Now using the assumed conditions, one can employ JensenSteffensen inequality given by Boas (see [29] or [30], p. 59) for convex function $R_{\mathrm{n}}(x, s)$ to obtain (6).

(ii) Since we can rewrite the R.H.S. of (7) in the difference

$$
H(\widetilde{\hbar})-\frac{\int_{a}^{b} H(\hbar(\zeta)) \mathrm{d} \lambda(\zeta)}{\int_{a}^{b} \mathrm{~d} \lambda(\zeta)}
$$

for convex function $H$ and by our assumed conditions on functions $\hbar$ and $\lambda$, this difference is non-positive by using Jensen-Steffensen inequality difference [29]. As a result, the R.H.S. of inequality (7) is non-positive and we get generalized Jensen-Steffensen inequality (2) for $\mathfrak{n}$-convex function.

Now, we give similar results related to Jensen-Boas inequality [30], p. 59], which is a generalization of Jensen-Steffensen inequality.

Corollary 1. Let $\Psi$ defined in Theorem 3 be $n$-convex function. Also, let $\hbar$ be as defined in $M_{1}$ with $a=y_{0}<y_{1}<\ldots<y_{k}<\ldots<y_{\mathrm{m}-1}<y_{\mathrm{m}}=b$ and $\hbar$ be monotonic in each of the $\mathfrak{m}$ intervals $\left(\left(y_{k-1}, y_{k}\right)\right)$. Then, the following results hold.

(i) If $\lambda$ as defined in $M_{2}$ satisfies

$$
\lambda(a) \leq \lambda\left(x_{1}\right) \leq \lambda\left(y_{1}\right) \leq \lambda\left(x_{2}\right) \leq \lambda\left(y_{2}\right) \leq \ldots \leq \lambda\left(y_{\mathfrak{m}-1}\right) \leq \lambda\left(x_{\mathfrak{m}}\right) \leq \lambda(b),
$$

$\forall x_{k} \in\left(y_{k-1}, y_{k}\right)$ and $\lambda(b)>\lambda(a)$, then for even $\mathfrak{n} \geq 3$, (6) is valid.

(ii) Moreover, if (6) is valid and the function $H(\cdot)$ defined in (18) is convex, then again inequality (2) holds and is called Jensen-Boas inequality for $\mathfrak{n}$-convex function.

Proof. We follow the similar argument as in the proof of Theorem 4, but under the conditions of this corollary, we utilize Jensen-Boas inequality (see [29] or [24], p. 59) instead of Jensen-Steffensen inequality.

Next, we give results for Jensen-Brunk inequality.

Corollary 2. Let $\Psi$ defined in Theorem 3 be $\mathfrak{n}$-convex and $\hbar$ defined in $M_{1}$ be an increasing function. Then, the following results hold.

(i) If $\lambda$ defined in $M_{2}$ with $\lambda(b)>\lambda(a)$ and

$$
\int_{a}^{x}(\hbar(x)-\hbar(\zeta)) \mathrm{d} \lambda(\zeta) \geq 0
$$

and

$$
\int_{x}^{b}(\hbar(x)-\hbar(\zeta)) \mathrm{d} \lambda(\zeta) \leq 0
$$

$\forall x \in[a, b]$ holds, then for even $\mathfrak{n} \geq 3$, (6) is valid.

(ii) Moreover, if (6) is valid and the function $H(\cdot)$ defined in (18) is convex, then again inequality (2) holds and is called Jensen-Brunk inequality for $\mathfrak{n}$-convex function.
Proof. We proceed with the similar idea as in the proof of Theorem 4, but under the conditions of this corollary, we employ Jensen-Brunk inequality (see [31] or [30], p. 59]) instead of Jensen-Steffensen inequality.

Remark 1. The similar result in Corollary 2 is also valid provided that the function $\hbar$ is decreasing. Also, assuming that the function $\hbar$ is monotonic, one can replace the conditions in Corollary 2(i) by

$$
0 \leq \int_{a}^{x}|\hbar(x)-\hbar(\zeta)| \mathrm{d} \lambda(\zeta) \leq \int_{x}^{b}|g(x)-\hbar(\zeta)| \mathrm{d} \lambda(\zeta) .
$$

Remark 2. It is interesting to see that by employing similar method as in Theorem 4, we can also get the generalization of classical Jensen's inequality (2) for $n$-convex functions by assuming the functions $\hbar$ and $\lambda$ along with the respective conditions in Theorem 1.

Another important consequence of Theorem 3 can be given by setting the function $\hbar$ as $\hbar(\zeta)=\zeta$. This form is the generalized version of L.H.S. inequality of the HermiteHadamard inequality.

Corollary 3. Let $\lambda:[a, b] \longrightarrow \mathbb{R}$ be a function of bounded variation such that $\lambda(a) \neq \lambda(b)$ with $[a, b] \subset[\alpha, \beta]$ and $\widetilde{\zeta}=\int_{a}^{b} \zeta d \lambda(\zeta) / \int_{a}^{b} d \lambda(\zeta) \in[\alpha, \beta]$. Under the assumptions of Theorem 3, if $\Psi$ is $\mathfrak{n}$-convex such that

$$
R_{\mathfrak{n}}(\tilde{\hbar}, s) \leq \frac{\int_{a}^{b} R_{\mathfrak{n}}(\hbar(\zeta), s) \mathrm{d} \lambda(\zeta)}{\int_{a}^{b} \mathrm{~d} \lambda(\zeta)}, \quad s \in[\alpha, \beta],
$$

then we have 


$$
\begin{aligned}
\Psi(\widetilde{\zeta}) \leq & \frac{\int_{a}^{b} \Psi(\zeta) \mathrm{d} \lambda(\zeta)}{\int_{a}^{b} \mathrm{~d} \lambda(\zeta)}+\sum_{\ell=0}^{\mathfrak{n}-2} \frac{1}{\ell !(\ell+2)(\beta-\alpha)} \\
& \times\left[\Psi^{(\ell+1)}(\alpha)\left((\widetilde{\zeta}-\alpha)^{(\ell+2)}-\frac{\int_{a}^{b}(\zeta-\alpha)^{(\ell+2)} \mathrm{d} \lambda(\zeta)}{\int_{a}^{b} \mathrm{~d} \lambda(\zeta)}\right)-\Psi^{(\ell+1)}(\beta)\left((\widetilde{\zeta}-\beta)^{(\ell+2)}+\frac{\int_{a}^{b}(\zeta-\beta)^{(\ell+2)} \mathrm{d} \lambda(\zeta)}{\int_{a}^{b} \mathrm{~d} \lambda(\zeta)}\right)\right]
\end{aligned}
$$

If the inequality (18) holds in reverse direction, then (19) also holds reversely.

The special case of above corollary can be given in the form of following remark.

Remark 3. It is interesting to see that substituting $\lambda(\zeta)=\zeta$ gives $\int_{a}^{b} \mathrm{~d} \lambda(\zeta)=b-a$ and $\widetilde{\zeta}=a+b / 2$. Using these substitutions in (2) and by following remark (20), we get the L.H.S. inequality of renowned Hermite-Hadamard inequality for $\mathfrak{n}$-convex functions.

2.2. New Generalization of Converse of Jensen's Integral Inequality. In this section, we give the results for the converse of Jensen's inequality to hold, giving the conditions on the real Stieltjes measure $\mathrm{d} \lambda$, such that $\lambda(a) \neq \lambda(b)$, allowing that the measure can also be negative, but employing Montgomery identity.

To start with we need the following assumption for the results of this section:

$A_{3} \quad$ Let $\mathbf{m}, \mathbf{M} \in[\alpha, \beta](\mathbf{m} \neq \mathbf{M})$ be such that $\mathbf{m} \leq \hbar(\zeta) \leq \mathbf{M}$ for all $\zeta \in[a, b]$ where $\hbar$ is defined in $A_{1}$.

For a given function $\Psi:[\alpha, \beta] \longrightarrow \mathbb{R}$, we consider the difference

$$
\mathrm{CJ}\left(\Psi, \hbar_{\{\mathbf{m}, \mathbf{M}\}} ; \lambda\right)=\frac{\int_{a}^{b} \Psi(\hbar(\zeta)) \mathrm{d} \lambda(\zeta)}{\int_{a}^{b} \mathrm{~d} \lambda(\zeta)}-\frac{\mathbf{M}-\tilde{\hbar}}{\mathbf{M}-\mathbf{m}} \Psi(\mathbf{m})-\frac{\tilde{\hbar}-\mathbf{m}}{\mathbf{M}-\mathbf{m}} \Psi(\mathbf{M}),
$$

where $\tilde{\hbar}$ is defined in (3).

$$
\operatorname{CJ}\left(R_{\mathfrak{n}}(x, s), \hbar_{\{\mathbf{m}, \mathbf{M}\}} ; \lambda\right) \leq 0, \quad s \in[\alpha, \beta],
$$

Using Montgomery identity, we obtain the following

or representation of the converse of Jensen's inequality.

Theorem 5. Let $\hbar, \lambda$ be as defined in $A_{1}, A_{2}$ and let $\Psi:[\alpha, \beta] \longrightarrow \mathbb{R}$ be such that for $\mathfrak{n} \geq 1, \Psi^{(\mathfrak{n}-1)}$ is absolutely continuous. If $\Psi$ is $\mathfrak{n}$-convex such that

$$
\frac{\int_{a}^{b} R_{\mathfrak{n}}(\hbar(\zeta), s) \mathrm{d} \lambda(\zeta)}{\int_{a}^{b} \mathrm{~d} \lambda(\zeta)} \leq \frac{\mathbf{M}-\widetilde{\hbar}}{\mathbf{M}-\mathbf{m}}\left(R_{\mathfrak{n}}(\mathbf{m}, s)\right)+\frac{\widetilde{\hbar}-\mathbf{m}}{\mathbf{M}-\mathbf{m}}\left(R_{\mathfrak{n}}(\mathbf{M}, s)\right), \quad s \in[\alpha, \beta],
$$

then we get the following extension of the converse of Jensen's

difference:

$$
\begin{aligned}
\frac{\int_{a}^{b} \Psi(\hbar(\zeta)) \mathrm{d} \lambda(\zeta)}{\int_{a}^{b} \mathrm{~d} \lambda(\zeta)} & \leq \frac{\mathbf{M}-\widetilde{\hbar}}{\mathbf{M}-\mathbf{m}} \Psi(\mathbf{m})+\frac{\widetilde{\hbar}-\mathbf{m}}{\mathbf{M}-\mathbf{m}} \Psi(\mathbf{M})+\sum_{\ell=0}^{\mathfrak{n}-2}\left(\frac{1}{\ell !(\ell+2)(\beta-\alpha)}\right) \\
& \times\left(\Psi^{(\ell+1)}(\alpha) \operatorname{CJ}\left((x-\alpha)^{\ell+2}, \hbar_{\{\mathbf{m}, \mathbf{M}\}} ; \lambda\right)-\Psi^{(\ell+1)}(\beta) \operatorname{CJ}\left((x-\beta)^{\ell+2}, \hbar_{\{\mathbf{m}, \mathbf{M}\}} ; \lambda\right)\right),
\end{aligned}
$$

where $R_{\mathfrak{n}}(\cdot, s)$ is defined in (5). 
Proof. As $\Psi^{(\mathfrak{n}-1)}$ is absolutely continuous for $(\mathfrak{n} \geq 1)$, we can use the representation of $\Psi$ using Montgomery identity (4) in the difference $\mathrm{CJ}\left(\Psi, \hbar_{\{\mathbf{m}, \mathbf{M}\}} ; \lambda\right)$ :

$$
\begin{aligned}
\mathrm{CJ}\left(\Psi, \hbar_{\{m, M\}} ; \lambda\right)= & \operatorname{CJ}\left(\frac{1}{\beta-\alpha} \int_{\alpha}^{\beta} \Psi(\zeta) \mathrm{d} \zeta, \hbar_{\{\mathbf{m}, \mathbf{M}\}} ; \lambda\right) \\
& +\sum_{\ell=0}^{\mathfrak{n}-2}\left(\frac{1}{\ell !(\ell+2)(\beta-\alpha)}\right) \Psi^{(\ell+1)}(\alpha) \operatorname{CJ}\left((x-\alpha)^{\ell+2}, \hbar_{\{\mathbf{m}, \mathbf{M}\}} ; \lambda\right) \\
& -\sum_{\ell=0}^{\mathfrak{n}-2}\left(\frac{1}{\ell !(\ell+2)(\beta-\alpha)}\right) \Psi^{(\ell+1)}(\beta) \operatorname{CJ}\left((x-\beta)^{\ell+2}, \hbar_{\{\mathbf{m}, \mathbf{M}\}} ; \lambda\right)+\frac{1}{(\mathfrak{n}-1) !} \int_{\alpha}^{\beta} \operatorname{CJ}\left(R_{\mathfrak{n}}(x, s), \hbar_{\{\mathbf{m}, \mathbf{M}\}} ; \lambda\right) \Psi^{(\mathfrak{n})}(s) \mathrm{d} s .
\end{aligned}
$$

After simplification and following the fact that $\mathrm{CJ}\left(\Psi, \hbar_{\{\mathbf{m}, \mathbf{M}\}} ; \lambda\right)$ is zero for $\Psi$ to be constant or linear, we get the following generalized identity:

$$
\begin{aligned}
\operatorname{CJ}\left(\Psi, \hbar_{\{\mathbf{m}, \mathbf{M}\}} ; \lambda\right) j= & \sum_{\ell=0}^{\mathfrak{n}-2}\left(\frac{\cdot}{\ell !(\ell+2)(\beta-\alpha)}\right) \\
& \times\left(\Psi^{(\ell+1)}(\alpha) \operatorname{CJ}\left((x-\alpha)^{\ell+2}, \hbar_{\{\mathbf{m}, \mathbf{M}\}} ; \lambda\right)-\Psi^{(\ell+1)}(\beta) \operatorname{CJ}\left((x-\beta)^{\ell+2}, \hbar_{\{\mathbf{m}, \mathbf{M}\}} ; \lambda\right)\right) \\
& +\frac{1}{(\mathfrak{n}-1) !} \int_{\alpha}^{\beta} \operatorname{CJ}\left(R_{\mathfrak{n}}(x, s), \hbar_{\{\mathbf{m}, \mathbf{M}\}} ; \lambda\right) \Psi^{(\mathfrak{n})}(s) \mathrm{d} s .(\text { CGI.1). }
\end{aligned}
$$

Now using characterizations of $\mathfrak{n}$-convex functions like in the proof of Theorem 3, we get (23).

The next result gives converse of Jensen's inequality for higher-order convex functions.

Theorem 6. Let $\Psi$ defined in Theorem 5 be $\mathfrak{n}$-convex and $\hbar$ be as defined in $A_{3}$. Then, the following results hold.

(i) If $\lambda$ is non-negative measure on $[a, b]$, then for even $\mathfrak{n} \geq 3$, (22) is valid.

(ii) Moreover, if (22) is valid and the function $H(\cdot)$ defined in (12) is convex, then we get the following inequality for $\mathfrak{n}$-convex function to be valid:

$$
\frac{\int_{a}^{b} \Psi(\hbar(\zeta)) \mathrm{d} \lambda(\zeta)}{\int_{a}^{b} \mathrm{~d} \lambda(\zeta)} \leq \frac{\mathbf{M}-\tilde{\hbar}}{\mathbf{M}-\mathbf{m}} \Psi(\mathbf{m})-\frac{\tilde{\hbar}-\mathbf{m}}{\mathbf{M}-\mathbf{m}} \Psi(\mathbf{M})
$$

Proof. The idea of the proof is similar to that of (6), but we use converse of Jensen's inequality (see [32] or [30], p. 98).

2.3. Applications of Jensen's Integral Inequality. In this section, we give applications of Jensen's integral inequality.

Another important consequence of Theorem 3 is by setting the function $\hbar$ as $\hbar(\zeta)=\zeta$ gives generalized version of L. H. S. inequality of the Hermite-Hadamard inequality.

Corollary 4. Let $\lambda:[a, b] \longrightarrow \mathbb{R}$ be a function of bounded variation such that $\lambda(a) \neq \lambda(b)$ with $[a, b] \subset[\alpha, \beta]$ and $\widetilde{\zeta}=\int_{a}^{b} \zeta d \lambda(\zeta) / \int_{a}^{b} d \lambda(\zeta) \in[\alpha, \beta]$. Under the assumptions of Theorem 5, if $\Psi$ is $\mathfrak{n}$-convex such that

$$
\frac{\int_{a}^{b} R_{\mathfrak{n}}(\zeta, s) \mathrm{d} \lambda(\zeta)}{\int_{a}^{b} \mathrm{~d} \lambda(\zeta)} \leq \frac{b-\tilde{\zeta}}{b-a}\left(R_{\mathfrak{n}}(a, s)\right)+\frac{\widetilde{\zeta}-a}{b-a}\left(R_{\mathfrak{n}}(b, s)\right), \quad s \in[\alpha, \beta],
$$

then we have 


$$
\begin{aligned}
& \frac{\int_{a}^{b} \Psi(\zeta) \mathrm{d} \lambda(\zeta)}{\int_{a}^{b} \mathrm{~d} \lambda(\zeta)} \leq \frac{b-\widetilde{\zeta}}{b-a} \Psi(a)+\frac{\widetilde{\zeta}-a}{b-a} \Psi(b)+\sum_{\ell=0}^{\mathfrak{n}-2}\left(\frac{.}{\ell !(\ell+2)(\beta-\alpha)}\right) \times \\
& \quad\left(\Psi^{(\ell+1)}(\alpha) \operatorname{CJ}\left((x-\alpha)^{\ell+2}, \operatorname{id}_{\{a, b\}} ; \lambda\right)-\Psi^{(\ell+1)}(\beta) \operatorname{CJ}\left((x-\beta)^{\ell+2}, i d_{\{a, b\}} ; \lambda\right)\right) .
\end{aligned}
$$

If the inequality (27) holds in reverse direction, then (28) also holds reversely.

The special case of above corollary can be given in the form of following remark.

Remark 4. It is interesting to see that substituting $\lambda(\zeta)=\zeta$ and by following Theorem 6 , we get the R.H.S. inequality of renowned Hermite-Hadamard inequality for $\mathfrak{n}$-convex functions.

\section{Generalization of Jensen's Discrete Inequality by Using Montgomery Identity}

In this section, we give generalizations for Jensen's discrete inequality by using Montgomery identity. The proofs are similar to those of continuous case as given in previous section; therefore, we give results directly.

3.1. Generalization of Jensen's Discrete Inequality for Real Weights. In discrete case, we have that $p_{q}>0$ for all $\mathscr{J}=1,2, \ldots, \mathfrak{m}$. Here we give generalizations of results allowing $p_{f}$ to be negative real numbers. Also, with usual notations for $p_{\mathscr{g}} x_{\mathscr{J}}(\mathscr{J}=1,2, \ldots, \mathfrak{n})$, we notate

$$
\mathbf{x}=\left(x_{1}, x_{2}, \ldots, x_{\mathfrak{m}}\right) \text { and } \mathbf{p}=\left(p_{1}, p_{2}, \ldots, p_{\mathfrak{m}}\right)
$$

to be $\mathfrak{m}$-tuples.

$$
P_{v}=\sum_{\mathcal{F}=1}^{v} p_{\mathcal{f}}, \bar{P}_{v}=P_{\mathfrak{m}}-P_{v-1} \quad(v=1,2, \ldots, \mathfrak{m}),
$$

and

$$
\bar{x}=\frac{1}{P_{\mathfrak{m}}} \sum_{\mathcal{g}=1}^{\mathfrak{m}} p_{\mathcal{g}} x_{\mathcal{F}}
$$

Using Montgomery identity (4), we obtain the following representations of Jensen's discrete inequality.

Theorem 7. Let $\Psi:[\alpha, \beta] \longrightarrow \mathbb{R}$ be such that for $\mathfrak{n} \geq 1$, $\Psi^{(\mathfrak{n}-1)}$ is absolutely continuous. Also, let $x_{g} \in[a, b] \subseteq[\alpha, \beta]$, $p_{\mathcal{F}} \in \mathbb{R}(\mathscr{J}=1, \ldots, \mathfrak{m})$ be such that $P_{\mathfrak{m}} \neq 0$ and $\bar{x} \in[\alpha, \beta]$.

(i) Then, the following generalized identity holds:

$$
\begin{aligned}
& \Psi(\bar{x})-\frac{1}{P_{\mathfrak{m}}} \sum_{\mathcal{f}=1}^{\mathfrak{m}} p_{\mathscr{f}} \Psi\left(x_{\mathcal{F}}\right)=\sum_{\ell=2}^{\mathfrak{n}-2}\left(\frac{1}{\ell !(\ell+2)(\beta-\alpha)}\right) \times \\
& \left\{\Psi^{(\ell+1)}(\alpha)\left((\bar{x}-\alpha)^{\ell+2}-\frac{1}{P_{\mathfrak{m}}} \sum_{\mathcal{g}=1}^{\mathfrak{m}} p_{\mathcal{J}}\left(x_{\mathcal{J}}-\alpha\right)^{\ell+2}\right)-\Psi^{(\ell+1)}(\beta)\left((\bar{x}-\beta)^{\ell+2}-\frac{1}{P_{\mathfrak{m}}} \sum_{\mathcal{J}=1}^{\mathfrak{m}} p_{\mathcal{g}}\left(x_{\mathcal{J}}-\beta\right)^{\ell+2}\right)\right\} \\
& +\frac{1}{(\mathfrak{n}-1) !} \int_{\alpha}^{\beta}\left[R_{\mathfrak{n}}(\bar{x}, s)-\frac{1}{P_{\mathfrak{m}}} \sum_{\mathcal{g}=1}^{\mathfrak{m}} p_{\mathcal{f}} R_{\mathfrak{n}}\left(x_{\mathscr{f}}, s\right)\right] \Psi^{(\mathfrak{n})}(s) \mathrm{d} s,(\text { DGI. } 1),
\end{aligned}
$$

where $R_{\mathfrak{n}}(\cdot, s)$ is defined in (5).

(ii) Moreover, if $\Psi$ is $\mathfrak{n}$-convex and the inequality

$$
R_{\mathfrak{n}}(\bar{x}, s) \leq \frac{1}{P_{\mathfrak{m}}} \sum_{\mathcal{f}=1}^{\mathfrak{m}} p_{\mathcal{f}} R_{\mathfrak{n}}\left(x_{\mathcal{f}}, s\right)
$$

holds, then we have the following generalized inequality:

$$
\begin{aligned}
\Psi(\bar{x})-\frac{1}{P_{\mathfrak{m}}} \sum_{\mathcal{F}=1}^{\mathfrak{m}} p_{\mathcal{f}} \Psi\left(x_{\mathcal{F}}\right) & \leq \sum_{\ell=2}^{\mathfrak{n}-2}\left(\frac{1}{\ell !(\ell+2)(\beta-\alpha)}\right) \times \\
& \left\{\Psi^{(\ell+1)}(\alpha)\left((\bar{x}-\alpha)^{\ell+2}-\frac{1}{P_{\mathfrak{m}}} \sum_{\mathcal{J}=1}^{\mathfrak{m}} p_{\mathcal{F}}\left(x_{\mathcal{F}}-\alpha\right)^{\ell+2}\right)-\Psi^{(\ell+1)}(\beta)\left((\bar{x}-\beta)^{\ell+2}-\frac{1}{P_{\mathfrak{m}}} \sum_{\mathcal{F}=1}^{\mathfrak{m}} p_{\mathcal{F}}\left(x_{\mathcal{F}}-\beta\right)^{\ell+2}\right)\right\} .
\end{aligned}
$$


If inequality (33) holds in reverse direction, then (34) also holds reversely.

Proof. Similar to that of Theorem 3.

In the later part of this section, we will vary our conditions on $p_{\mathcal{F}} x_{\mathscr{f}}(\mathscr{J}=1,2, \ldots, \mathfrak{n})$ to obtain generalized discrete variants of Jensen-Steffensen, Jensen's, and Jensen-Petrovic type inequalities. We start with the following generalization of Jensen-Steffensen discrete inequality for $\mathfrak{n}$-convex functions.

Theorem 8. Let $\Psi$ be as defined in Theorem 7. Also, let $\mathbf{x}$ be monotonic $\mathfrak{n}$-tuple, $x_{g} \in[a, b] \subseteq[\alpha, \beta]$, and $\mathbf{p}$ be a real $\mathfrak{n}$-tuple such that

$$
0 \leq P_{v} \leq P_{\mathfrak{m}},(v=1,2, \ldots, \mathfrak{m}-1), \quad P_{\mathfrak{m}}>0
$$

is satisfied.

(i) If $\Psi$ is $\mathfrak{n}$-convex, then for even $\mathfrak{n} \geq 3$, (33) is valid.

(ii) Moreover, if (33) is valid and the function $H(\cdot)$ defined in (12) is convex, then we get the following generalized Jensen-Steffensen discrete inequality:

$$
\Psi(\bar{x}) \leq \frac{1}{P_{\mathfrak{m}}} \sum_{\mathscr{J}=1}^{\mathfrak{m}} p_{\mathscr{J}} \Psi\left(x_{\mathscr{J}}\right)
$$

Proof. It is interesting to see that under the assumed conditions on tuples $\mathbf{x}$ and $\mathbf{p}$, we have that $\bar{x} \in[a, b]$. For $x_{1} \geq x_{2} \geq \ldots \geq x_{\mathrm{m}}$,

$$
P_{\mathfrak{m}}\left(x_{1}-\bar{x}\right)=\sum_{\mathcal{F}=2}^{\mathfrak{m}} p_{\mathcal{J}}\left(x_{1}-x_{\mathcal{J}}\right)=\sum_{v=2}^{\mathfrak{m}}\left(x_{v-1}-x_{v}\right)\left(P_{\mathfrak{m}}-P_{v-1}\right) \geq 0 .
$$

This shows that $x_{1} \geq \bar{x}$. Also, $\bar{x} \geq x_{\mathfrak{n}}$, since we have

$$
P_{\mathfrak{m}}\left(\bar{x}-x_{\mathfrak{m}}\right)=\sum_{\mathcal{J}=1}^{\mathfrak{m}-1} p_{\mathcal{J}}\left(x_{\mathcal{f}}-x_{\mathfrak{m}}\right)=\sum_{v=1}^{\mathfrak{m}-1}\left(x_{v}-x_{v-1}\right) P_{v} \geq 0 .
$$

For further details, see the proof of Jensen-Steffensen discrete inequality ([24], p. 57). The idea of the rest of the proof is similar to that of Theorem 3, but here we employ Theorem 7 and Jensen-Steffensen discrete inequality.

Corollary 5. Let $\Psi$ be as defined in Theorem 7 and let $x_{\mathscr{g}} \in[a, b] \subseteq[\alpha, \beta]$ with $\mathbf{p}$ being a positive $\mathfrak{n}$-tuple.

(i) If $\Psi$ is $\mathfrak{n}$-convex, then for even $\mathfrak{n} \geq 3$, (34) is valid.

(ii) Moreover, if (33) is valid and the function $H($.$) de-$ fined in (12) is convex, then again we get (36) which is called Jensen's inequality for $\mathfrak{n}$-convex functions.

Proof. For $p_{\mathscr{J}}>0, x_{\mathscr{J}} \in[a, b](\mathscr{J}=1,2,3, \ldots, \mathfrak{m})$ ensures that $\bar{x} \in[a, b]$. So, by applying classical Jensen's discrete inequality (1) and idea of Theorem 8, we will get the required results.

Remark 5. Under the assumptions of Corollary 5, if we choose $P_{\mathfrak{m}}=1$, then Corollary 5 (ii) gives the following inequality for $\mathfrak{n}$-convex functions:

$$
\Psi\left(\sum_{\mathcal{I}=1}^{\mathfrak{m}} p_{\mathscr{J}} x_{\mathcal{J}}\right) \leq \sum_{\mathcal{J}=1}^{\mathfrak{m}} p_{\mathscr{J}} \Psi\left(x_{\mathcal{F}}\right) .
$$

Now we give following reverses of Jensen-Steffensen and Jensen-type inequalities.

Corollary 6. Let $\Psi$ be as defined in Theorem 7. Also, let $\mathbf{x}$ be monotonic $\mathfrak{m}$-tuple, $x_{g} \in[a, b] \subseteq[\alpha, \beta]$, and $\mathbf{p}$ be a real $\mathfrak{m}$-tuple such that there exist $m \in\{1,2, \ldots, \mathfrak{m}\}$ such that

$$
0 \geq P_{v}, \quad \text { for } v<m \text { and } 0 \geq \bar{P}_{v} \text {, for } v>m,
$$

where $P_{\mathfrak{m}}>0$ and $\bar{x} \in[\alpha, \beta]$.

(i) If is $\mathfrak{n}$-convex, then for even $\mathfrak{n} \geq 3$, then reverse of inequality (33) holds.

(ii) Moreover if (33) holds reversely and the function $H(\cdot)$ defined in (12) is convex, then we get reverse of generalized Jensen-Steffensen inequality (36) for $\mathfrak{n}$-convex functions.

Proof. We follow the idea of Theorem 8, but according to our assumed conditions, we employ reverse of JensenSteffensen inequality to obtain results.

In the next corollary, we give explicit conditions on real tuple $\mathbf{p}$ such that we get reverse of classical Jensen inequality.

Corollary 7. Let $\Psi$ be as defined in Theorem 7 and let $x_{g} \in[a, b] \subseteq[\alpha, \beta]$ such that $\bar{x} \in[\alpha, \beta]$. Let $\mathbf{p}$ be a real $n-t u p l e$ such that

$$
0<p_{1}, 0 \geq p_{2}, p_{3}, \ldots, p_{\mathfrak{m}}, 0<P_{\mathfrak{m}}
$$

is satisfied.

(i) If $\Psi$ is $\mathfrak{n}$-convex, then for even $\mathfrak{n} \geq 3$, the reverse of inequality (33) is valid.

(ii) Also, if reverse of (33) is valid and the function $H(\cdot)$ defined in (12) is convex, then we get reverse of (36).

Proof. We follow the idea of Theorem 8, but according to our assumed conditions, we employ reverse of Jensen inequality to obtain results.

In [33] (see also [30]), one can find the result which is equivalent to the Jensen-Steffensen and the reverse Jensen-Steffensen inequality together. It is the so-called Jensen-Petrović inequality. Here, without the proof, we give the adequate corollary which uses that result. The proof goes the same way as in the previous corollaries. 
Corollary 8. Let $\Psi$ be as defined in Theorem 7 and let $x_{i} \in[a, b] \subseteq[\alpha, \beta]$ be such that $x_{\mathfrak{m}} \geq x_{\mathfrak{m}-1}, \ldots, x_{2} \leq x_{1}$. Let $\mathbf{p}$ be a real $\mathfrak{m}$-tuple with $P_{\mathfrak{m}}=1$ such that

$$
0 \leq P_{v}, \quad \text { for } 1 \leq v<\mathfrak{m}-1 \text { and } 0 \leq \bar{P}_{v}, \quad \text { for } 2 \leq v<\mathfrak{m},
$$

is satisfied. Then, we get the equivalent results given in Theorem 8 ( $i$ ) and (ii), respectively.

Remark 6. Under the assumptions of Corollary 8, if there exist $m \in\{1,2, \ldots, \mathfrak{n}\}$ such that

$$
0 \geq P_{v}, \quad \text { for } v<m \text { and } 0 \geq \bar{P}_{v}, \quad \text { for } v>m,
$$

and $\bar{x} \in[\alpha, \beta]$, then we get the equivalent results for reverse Jensen-Steffensen inequality given in Corollary 6 (i) and (ii), respectively.

Remark 7. It is interesting to see that the conditions on $p_{\mathcal{f}}, \mathscr{J}=1,2, \ldots, \mathfrak{m}$ given in Corollary 8 and Remark 6 are coming from Jensen-Petrović inequality which become equivalent to conditions for $p_{\mathscr{g}}, \mathcal{J}=1,2, \ldots, \mathfrak{m}$ for Jensen-Steffensen results given in Theorem 8 and Corollary 6 , respectively, when $P_{\mathrm{m}}=1$.

Now we give results for Jensen and its reverses for $\mathfrak{n}$-tuples $\mathbf{x}$ and $\mathbf{p}$ when $\mathfrak{n}$ is an odd number.

Corollary 9. Let $\Psi$ be as defined in Theorem 7 and let $x_{\mathscr{g}} \in[a, b] \subseteq[\alpha, \beta]$ for $\mathscr{J}=1,2, \ldots, \mathfrak{m}$ be such that $\mathbf{x}, \mathbf{p}$ be real $\mathfrak{m}$-tuples, $\mathfrak{m}=2 m+1, m \in \mathbb{N}$ and $\hat{x}=1 / \sum_{\mathcal{f}=1}^{2 k+1} p_{\mathcal{g}} \sum_{\mathcal{f}=1}^{2 k+1}$ $p_{\mathcal{g}} x_{\mathcal{g}} \in[\alpha, \beta]$ for all $k=1,2, \ldots, m$. If for every $k=1,2, \ldots, m$, we have

$$
\begin{aligned}
& \left(i^{*}\right) p_{1}>0, p_{2 k} \leq 0, p_{2 k}+p_{2 k+1} \leq 0, \quad \sum_{\mathcal{J}=1}^{2 k} p_{\mathcal{J}} \geq 0, \\
& \sum_{\mathscr{J}=1}^{2 k+1} p_{\mathscr{J}}>0 \\
& \text { (ii } \left.i^{*}\right) x_{2 k} \leq x_{2 k+1}, \sum_{\mathcal{f}=1}^{2 k+1} p_{\mathcal{F}}\left(x_{\mathcal{g}}-x_{2 k+1}\right) \geq 0 \text {, }
\end{aligned}
$$

then we have the following statements to be valid.

(i) If $\Psi$ is $\mathfrak{n}$-convex, then for even $\mathfrak{n} \geq 3$, the inequality

$$
R_{\mathfrak{n}}(\widehat{x}, s) \geq \frac{1}{P_{2 m+1}} \sum_{\mathscr{J}=1}^{2 m+1} p_{\mathscr{J}} R_{\mathfrak{n}}\left(x_{\mathscr{J}}, s\right) .
$$

(ii) Also if (44) is valid and the function $H(\cdot)$ defined in (12) is convex, then we get the following generalized inequality:

$$
\Psi(\widehat{x}) \geq \frac{1}{P_{2 m+1}} \sum_{\mathcal{J}=1}^{2 m+1} p_{\mathscr{f}} \Psi\left(x_{\mathscr{f}}\right)
$$

Proof. We employ the idea of the proofs of Theorems 7 and 8 for $\mathfrak{n}=$ odd along with inequality of Vasić and Janic [34].

Remark 8. We can also discuss the following important cases by considering the explicit conditions given in [34].

We conclude this section by giving the following important cases:

\section{(Case 1)}

Let the condition $\left(i^{*}\right)$ hold and the reverse inequalities in condition $\left(i i^{*}\right)$ hold. Then, again we can give inequalities (44) and (45), respectively, given in Corollary 9.

(Case 2)

If in case of conditions $\left(i^{*}\right)$ and $\left(i i^{*}\right)$, the following are valid:

$\left(i i i^{*}\right) \quad p_{1}>0, p_{2 k+1} \geq 0, p_{2 k}+p \quad 2 k+1 \geq 0, \sum_{\mathcal{I}=1}^{2 k} p_{\mathscr{g}} \geq 0$, $\sum_{\mathscr{J}=1}^{2 k+1} p_{\mathscr{J}}>0$

$\left(i v^{*}\right) x_{2 k} \leq x_{2 k+1}, \sum_{\mathcal{J}=1}^{2 k-1} p_{\mathcal{J}}\left(x_{\mathcal{J}}-x_{2 k}\right) \leq 0$,

then we can give reverses of inequalities (44) and (45), respectively, given in Corollary 9.

\section{(Case 3)}

Finally, we can also give reverses of inequalities (44) and (45), respectively, given in Corollary 9 provided that the condition $\left(\mathrm{iii}^{*}\right)$ holds and the reverse inequalities in condition $\left(i v^{*}\right)$ hold.

The result given in (Case 3) is type of generalization of inequality by Szegö [35].

3.2. Generalization of Converse Jensen's Discrete Inequality for Real Weights. In this section, we give the results for converse of Jensen's inequality in discrete case by using the Montgomery identity.

Let $x_{g} \in[a, b] \subseteq[\alpha, \beta], a \neq b, p_{g} \in \mathbb{R}(\mathscr{J}=1, \ldots, \mathfrak{n})$ be such that $P_{\mathfrak{m}} \neq 0$. Then, we have the following difference of converse of Jensen's inequality for $\Psi:[\alpha, \beta] \longrightarrow \mathbb{R}$ :

$$
\mathrm{CJ}_{\mathrm{dis}}(\Psi)=\frac{1}{P_{\mathfrak{m}}} \sum_{\mathcal{J}=1}^{\mathfrak{m}} p_{\mathcal{F}} \Psi\left(x_{\mathcal{J}}\right)-\frac{b-\bar{x}}{b-a} \Psi(a)-\frac{\bar{x}-a}{b-a} \Psi(b) .
$$

Similarly, we assume the Giaccardi difference [36] given as

$$
G_{\text {cardi }}(\Psi)=\sum_{\mathcal{F}=1}^{\mathfrak{m}} p_{\mathcal{f}} \Psi\left(x_{\mathcal{f}}\right)-A \Psi\left(\sum_{\mathcal{F}=1}^{\mathfrak{m}} p_{\mathcal{F}} x_{\mathcal{F}}\right)-B\left(\sum_{\mathcal{F}=1}^{\mathfrak{m}} p_{\mathcal{F}}-1\right) \Psi\left(x_{0}\right),
$$

where 


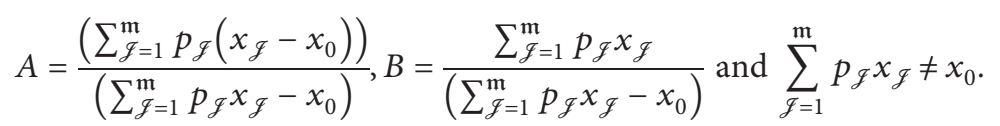

Theorem 9. Let $\Psi:[\alpha, \beta] \longrightarrow \mathbb{R}$ be such that for $\mathfrak{n} \geq 1$, $\Psi^{(\mathfrak{n}-1)}$ is absolutely continuous. Also, let $x_{0}, x_{\mathscr{g}} \in[a, b] \subseteq[\alpha, \beta], p_{\mathscr{g}} \in \mathbb{R}(\mathscr{J}=1, \ldots, \mathfrak{m})$, be such that $\sum_{\mathcal{F}=1}^{\mathfrak{m}} p_{\mathcal{f}} x_{\mathcal{F}} \neq x_{0}$. (i) Then, the following generalized identity holds:

$$
\begin{aligned}
& \mathrm{CJ}_{\mathrm{dis}}(\Psi)= \sum_{\ell=0}^{\mathfrak{n}-2}\left(\frac{1}{\ell !(\ell+2)(\beta-\alpha)}\right)\left(\Psi^{(\ell+1)}(\alpha) \mathrm{CJ}_{\mathrm{dis}}\left(\left(x_{\mathcal{F}}-\alpha\right)^{\ell+2}\right)-\Psi^{(\ell+1)}(\beta) \mathrm{CJ}_{\mathrm{dis}}\left(\left(x_{\mathscr{f}}-\beta\right)^{\ell+2}\right)\right) \\
&+\frac{1}{(\mathfrak{n}-1) !} \int_{\alpha}^{\beta} \mathrm{CJ}_{\mathrm{dis}}\left(R_{\mathfrak{n}}\left(x_{\mathcal{F}}, s\right)\right) \Psi^{(\mathfrak{n})}(s) \mathrm{d} s,(\text { DC.GI }), \\
& \quad \mathrm{CJ}_{\operatorname{dis}}\left(R_{\mathfrak{n}}\left(x_{\mathscr{f}}, s\right)\right) \leq 0
\end{aligned}
$$

where $R_{\mathfrak{n}}(\cdot, s)$ is defined in (5).

(ii) Moreover, if $\Psi$ is $\mathfrak{n}$-convex and the inequality holds, then we have the following generalized inequality:

$$
\mathrm{CJ}_{\mathrm{dis}}(\Psi) \leq \sum_{\ell=0}^{\mathfrak{n}-2}\left(\frac{1}{\ell !(\ell+2)(\beta-\alpha)}\right)\left(\Psi^{(\ell+1)}(\alpha) \mathrm{C} \mathrm{J}_{\mathrm{dis}}\left(\left(x_{\mathscr{g}}-\alpha\right)^{\ell+2}\right)-\Psi^{(\ell+1)}(\beta) \mathrm{CJ} \operatorname{dis}\left(\left(x_{\mathscr{F}}-\beta\right)^{\ell+2}\right)\right) .
$$

If inequality (50) holds in reverse direction, then (51) also holds reversely. (i) Then, the following generalized Giaccardi identity holds:

Theorem 10. Let $\Psi:[\alpha, \beta] \longrightarrow \mathbb{R}$ be such that for $\mathfrak{n} \geq 1$, $\Psi^{(\mathfrak{n}-1)}$ is absolutely continuous. Also, let $x_{f} \in[a, b] \subseteq[\alpha, \beta]$, $p_{\mathscr{g}} \in \mathbb{R}(\mathscr{J}=1, \ldots, \mathfrak{m})$, be such that $P_{\mathfrak{m}} \neq 0$ and $\bar{x} \in[\alpha, \beta]$.

$$
\begin{aligned}
G_{\text {cardi }}(\Psi)= & \sum_{\ell=0}^{\mathfrak{n}-2}\left(\frac{1}{\ell !(\ell+2)(\beta-\alpha)}\right)\left(\Psi^{(\ell+1)}(\alpha) G_{\text {cardi }}\left(\left(x_{\mathscr{F}}-\alpha\right)^{\ell+2}\right)-\Psi^{(\ell+1)}(\beta) G_{\text {cardi }}\left(\left(x_{\mathscr{J}}-\beta\right)^{\ell+2}\right)\right) \\
& +\frac{1}{(\mathfrak{n}-1) !} \int_{\alpha}^{\beta} G_{\text {cardi }}\left(R_{\mathfrak{n}}\left(x_{\mathscr{f}}, s\right)\right) \Psi^{(\mathfrak{n})}(s) \mathrm{d} s, \text { (GIA.GI) }
\end{aligned}
$$

where $R_{\mathfrak{n}}(\cdot, s)$ is defined in (5).

(ii) Moreover, if $\Psi$ is $\mathfrak{n}$-convex and the inequality

$$
G_{\text {cardi }}\left(R_{\mathfrak{n}}\left(x_{\mathscr{f}}, s\right)\right) \leq 0
$$

holds, then we have the following generalized Giaccardi inequality:

$$
G_{\text {cardi }}(\Psi) \leq \sum_{\ell=0}^{\mathfrak{n}-2}\left(\frac{1}{\ell !(\ell+2)(\beta-\alpha)}\right)\left(\Psi^{(\ell+1)}(\alpha) G_{\text {cardi }}\left(\left(x_{\mathscr{f}}-\alpha\right)^{\ell+2}\right)-\Psi^{(\ell+1)}(\beta) G_{\text {cardi }}\left(\left(x_{\mathscr{F}}-\beta\right)^{\ell+2}\right)\right) .
$$

If inequality (53) holds in reverse direction, then (54) also holds reversely.
In the later part of this section, we will vary our conditions on $p_{\mathscr{f}} x_{\mathcal{F}}(\mathscr{J}=1,2, \ldots, \mathfrak{m})$ to obtain generalized 
converse discrete variants of Jensen's inequality and Giaccardi inequality for $\mathfrak{n}$-convex functions.

Theorem 11. Let $\Psi$ be as defined in Theorem 9. Also, let $x_{g} \in[a, b] \subseteq[\alpha, \beta]$ and $\mathbf{p}$ be a positive $\mathfrak{m}$-tuple.

(i) If $\Psi$ is $\mathfrak{n}$-convex, then for even $\mathfrak{n} \geq 3$, (50) is valid.

(ii) Moreover, if (50) is valid and the function $H(\cdot)$ defined in (12) is convex, then we get the following generalized converse of Jensen's inequality:

$$
\frac{1}{P_{\mathfrak{m}}} \sum_{\mathcal{F}=1}^{\mathfrak{m}} p_{\mathcal{F}} \Psi\left(x_{\mathcal{F}}\right) \leq \frac{b-\bar{x}}{b-a} \Psi(a)+\frac{\bar{x}-a}{b-a} \Psi(b) .
$$

Proof. We follow the idea of Theorem 8, but according to our assumed conditions, we employ converse of Jensen's inequality (see [32] or [30], p. 98) to obtain results.

Finally, in this section, we give Giaccardi inequality for higher-order convex functions.

Theorem 12. Let $\Psi$ be as defined in Theorem 9. Also, let $x_{0}, x_{\mathcal{g}} \in[a, b] \subseteq[\alpha, \beta]$ and $\mathbf{p}$ be a positive $\mathbf{m}$-tuple such that

$$
\sum_{\mathcal{J}=1}^{\mathfrak{m}} p_{\mathcal{g}} x_{\mathcal{J}} \neq x_{0} \text { and }\left(x_{v}-x_{0}\right)\left(\sum_{\mathcal{F}=1}^{\mathfrak{m}} p_{\mathcal{F}} x_{\mathcal{F}}-x_{v}\right) \geq 0, \quad(v=1, \ldots, \mathfrak{m})
$$

(i) If $\Psi$ is $\mathfrak{n}$-convex, then for even $\mathfrak{n} \geq 3$, (53) is valid.

(ii) Moreover, if (53) is valid and the function $H(\cdot)$ defined in (12) is convex, then we get the following generalized Giaccardi inequality:

$$
\sum_{\mathcal{J}=1}^{\mathfrak{m}} p_{\mathscr{J}} \Psi\left(x_{\mathcal{F}}\right) \leq A \Psi\left(\sum_{\mathcal{J}=1}^{\mathfrak{m}} p_{\mathscr{J}} x_{\mathcal{F}}\right)+B\left(\sum_{\mathcal{F}=1}^{\mathfrak{m}} p_{\mathscr{J}}-1\right) \Psi\left(x_{0}\right)
$$

where $A$ and $B$ are defined in (47).

Proof. We follow the idea of Theorem 8, but according to our assumed conditions, we employ Giaccardi inequality (see [36] or [37], p. 11) to obtain results.

3.3. Applications in Information Theory for Jensen's Discrete Inequality. Jensen's inequality plays a key role in information theory to construct lower bounds for some notable inequalities, but here we will use it to make connections between inequalities in information theory.

Let $\Psi: \mathbb{R}^{+} \longrightarrow \mathbb{R}^{+}$be a convex function and let $p:=\left(p_{1}, \ldots, p_{\mathfrak{m}}\right)$ and $q:=\left(q_{1}, \ldots, q_{\mathfrak{m}}\right)$ be positive probability distributions; then, $\Psi$-divergence functional is defined (in [38]) as follows:

$$
I_{\Psi}(\mathbf{p}, \mathbf{q})=\sum_{\mathcal{J}=1}^{\mathfrak{m}} q_{\mathscr{J}} \Psi\left(\frac{p_{\mathscr{J}}}{q_{\mathscr{J}}}\right) .
$$

Horváth et al. in [39] defined the generalized Csiszár divergence functional as follows.

Definition 2. Let $I$ be an interval in $\mathbb{R}$ and $\Psi: I \longrightarrow \mathbb{R}$ be a function. Also, let $\mathbf{p}:=\left(p_{1}, \ldots, p_{\mathfrak{m}}\right) \in \mathbb{R}^{\mathfrak{m}}$ and $\mathbf{q}:=\left(q_{1}, \ldots, q_{\mathfrak{m}}\right) \in(0, \infty)^{\mathfrak{m}}$ such that

$$
\frac{p_{\mathscr{g}}}{q_{\mathscr{J}}} \in I, \quad \mathcal{J}=1, \ldots, \mathfrak{m} .
$$

Then, let

$$
\widetilde{I}_{\Psi}(\mathbf{p}, \mathbf{q})=\sum_{\mathcal{J}=1}^{\mathfrak{m}} q_{\mathscr{f}} \Psi\left(\frac{p_{\mathscr{g}}}{q_{\mathcal{F}}}\right)
$$

In this section, we write Jensen's difference here that we use in upcoming results:

$$
F\left(\mathbf{p}, x_{\mathscr{f}}, \Psi\right)=\Psi(\bar{x})-\frac{1}{P_{\mathfrak{m}}} \sum_{\mathcal{F}=1}^{\mathfrak{m}} p_{\mathscr{f}} \Psi\left(x_{\mathscr{f}}\right) .
$$

Theorem 13. Under the assumptions of Theorem 9 (ii), let (51) hold and $\Psi$ be $\mathfrak{n}$-convex. Also, let $\mathbf{p}:=\left(p_{1}, \ldots, p_{\mathfrak{m}}\right)$ in $\mathbb{R}^{\mathfrak{m}}$ and $\mathbf{q}:=\left(q_{1}, \ldots, q_{\mathfrak{m}}\right) \in(0, \infty)^{\mathfrak{m}}$; then, we have the following results: 


$$
\tilde{I}_{\Psi}(\mathbf{p}, \mathbf{q}) \geq P_{\mathfrak{m}} \Psi(1)-P_{\mathfrak{m}} \sum_{\ell=2}^{\mathfrak{n}-2}\left(\frac{1}{\ell !(\ell+2)(\beta-\alpha)}\right) \times\left\{\Psi^{(\ell+1)}(\alpha)\left[F\left(\mathbf{q}, \frac{p_{\mathcal{g}}}{q_{\mathcal{g}}},(x-\alpha)^{\ell+2}\right)\right]-\Psi^{(\ell+1)}(\beta)\left[F\left(\mathbf{q}, \frac{p_{\mathcal{g}}}{q_{\mathcal{g}}},(x-\beta)^{\ell+2}\right)\right]\right\} .
$$

Proof. From Theorem 9 by following Jensen's difference (61), we can rearrange (34) as

$$
\Psi(\bar{x})-\frac{1}{P_{\mathfrak{m}}} \sum_{\mathcal{J}=1}^{\mathfrak{m}} p_{\mathcal{J}} \Psi\left(x_{\mathcal{F}}\right) \leq \sum_{\ell=2}^{\mathfrak{n}-2}\left(\frac{1}{\ell !(\ell+2)(\beta-\alpha)}\right) \times\left\{\Psi^{(\ell+1)}(\alpha)\left(F\left(\mathbf{p}, x_{\mathscr{f}},(x-\alpha)^{\ell+2}\right)\right)-\Psi^{(\ell+1)}(\beta)\left(F\left(\mathbf{p}, x_{\mathcal{F}},(x-\beta)^{\ell+2}\right)\right)\right\} .
$$

Now replace $p_{\mathscr{f}}$ with $q_{\mathscr{f}}$ and $x_{\mathscr{f}}$ with $p_{\mathscr{f}} / q_{\mathscr{f}}$, and we get (62).

For positive $\mathfrak{n}$-tuple $\mathbf{q}=\left(q_{1}, \ldots, q_{\mathfrak{m}}\right)$ such that $\sum_{\mathcal{F}=1}^{\mathfrak{m}} q_{\mathcal{F}}=1$, the Shannon entropy is defined by

$$
S(q)=-\sum_{\mathcal{g}=1}^{\mathfrak{m}} q_{\mathcal{g}} \ln q_{\mathcal{f}} .
$$

Corollary 10. Under the assumptions of Theorem 9 (ii), let (51) hold and $\Psi$ be $\mathfrak{n}$-convex.

(i) If $\mathbf{q}:=\left(q_{1}, \ldots, q_{\mathfrak{m}}\right) \in(0, \infty)^{\mathfrak{m}}$, then

$$
\sum_{\mathcal{g}=1}^{\mathfrak{m}} q_{\mathcal{g}} \ln q_{\mathcal{g}} \leq P_{\mathfrak{m}} \sum_{\ell=2}^{\mathfrak{n}-2}\left(\frac{1}{\ell !(\ell+2)(\beta-\alpha)}\right) \times\left\{\frac{(-1)^{\ell+1} \ell !}{\alpha^{(\ell+1)}} F\left(q, \frac{1}{q_{\mathcal{g}}},-\ln (\cdot)\right)-\frac{(-1)^{\ell+1} \ell !}{\beta^{(\ell+1)}} F\left(q, \frac{1}{q_{\mathcal{g}}},-\ln (\cdot)\right)\right\} .
$$

(ii) We can get bounds for the Shannon entropy of $\mathbf{q}$, if we choose $\mathbf{q}:=\left(q_{1}, \ldots, q_{\mathfrak{n}}\right)$ to be a positive probability distribution.

$$
S(\mathbf{q}) \leq P_{\mathfrak{m}} \sum_{\ell=2}^{\mathfrak{n}-2}\left(\frac{1}{\ell !(\ell+2)(\beta-\alpha)}\right) \times\left\{\frac{(-1)^{\ell+1} \ell !}{\alpha^{(\ell+1)}} F\left(\mathbf{q}, \frac{1}{q_{\mathcal{g}}},-\ln (\cdot)\right)-\frac{(-1)^{\ell+1} \ell !}{\beta^{(\ell+1)}} F\left(\mathbf{q}, \frac{1}{q_{\mathcal{J}}},-\ln (\cdot)\right)\right\} .
$$

Proof. (i) Substituting $\Psi(x):=-\ln x$ and using $\mathbf{p}:=(1,1, \ldots, 1)$ in Theorem 13, we get (65).

$$
D(\mathbf{q} \| \mathbf{p})=\sum_{\mathcal{g}=1}^{\mathfrak{m}} q_{\mathcal{g}} \ln \left(\frac{q_{\mathcal{g}}}{p_{\mathscr{f}}}\right) .
$$

(ii) Since we have $\sum_{\mathcal{q}=1}^{\mathfrak{m}} q_{q}=1$, by multiplying -1 on both sides of (65) and taking into account (64), we get (66).

The Kullback-Leibler distance [40] between the positive probability distributions $\mathbf{p}=\left(p_{1}, \ldots, p_{\mathfrak{m}}\right)$ and

Corollary 11. Under the assumptions of Corollary 10,

(i) If $\mathbf{q}:=\left(q_{1}, \ldots, q_{\mathfrak{m}}\right), \mathbf{p}:=\left(p_{1}, \ldots, p_{\mathfrak{m}}\right) \in(0, \infty)^{\mathfrak{m}}$, then $\mathbf{q}=\left(q_{1}, \ldots, q_{\mathfrak{m}}\right)$ is defined by

$$
\sum_{\mathcal{F}=1}^{\mathfrak{m}} q_{\mathcal{g}} \ln \left(\frac{q_{\mathcal{I}}}{p_{\mathcal{g}}}\right) \leq P_{\mathfrak{m}} \sum_{\ell=2}^{\mathfrak{n}-2}\left(\frac{1}{\ell !(\ell+2)(\beta-\alpha)}\right) \times\left\{\frac{(-1)^{\ell+1} \ell !}{\alpha^{(\ell+1)}} F\left(q, \frac{p_{\mathcal{J}}}{q_{\mathcal{J}}},-\ln (\cdot)\right)-\frac{(-1)^{\ell+1} \ell !}{\beta^{(\ell+1)}} F\left(q, \frac{p_{\mathcal{g}}}{q_{\mathcal{g}}},-\ln (\cdot)\right)\right\} .
$$

(ii) If $\mathbf{q}:=\left(q_{1}, \ldots, q_{\mathfrak{m}}\right), \mathbf{p}:=\left(p_{1}, \ldots, p_{\mathfrak{m}}\right)$ are positive probability distributions, then we have 


$$
D(\mathbf{q} \| \mathbf{p}) \leq P_{\mathfrak{m}} \sum_{\ell=2}^{\mathfrak{n}-2}\left(\frac{1}{\ell !(\ell+2)(\beta-\alpha)}\right) \times\left\{\frac{(-1)^{\ell+1} \ell !}{\alpha^{(\ell+1)}} F\left(q, \frac{p_{\mathscr{J}}}{q_{\mathcal{F}}},-\ln (\cdot)\right)-\frac{(-1)^{\ell+1} \ell !}{\beta^{\ell+1}} F\left(q, \frac{p_{\mathscr{F}}}{q_{\mathcal{J}}},-\ln (\cdot)\right)\right\} .
$$

Proof.

(i) Using $\Psi(x):=-\ln x$ (which is $\mathfrak{n}$-convex for even $\mathfrak{n}$ ) in Theorem 13, we get (68) after simplification.

(ii) It is a special case of (i).

3.4. Results for Zipf and Hybrid Zipf-Mandelbrot Entropy. One of the basic laws in information science is Zipf's law $[41,42]$ which is highly applied in linguistics. Let $c \geq 0, d>0$, and $N \in\{1,2, \ldots\} ;$ Zipf-Mandelbrot entropy can be given as

$$
Z_{M}(H, c, d)=\frac{d}{H_{c, d}^{N}} \sum_{\mathscr{J}=1}^{N} \frac{\ln (\mathscr{J}+c)}{(\mathscr{J}+c)^{d}}+\ln \left(H_{c, d}^{N}\right),
$$

where

$$
H_{c, d}^{N}=\sum_{\sigma=1}^{N} \frac{1}{(\sigma+c)^{d}}
$$

\section{Consider}

$$
q_{\mathscr{g}}=\Psi(\mathscr{J} ; N, c, d)=\frac{1}{\left((\mathscr{J}+c)^{d} H_{c, d}^{N}\right)},
$$

where $\Psi(\mathscr{J} ; m, c, d)$ is discrete probability distribution known as Zipf-Mandelbrot law. Zipf-Mandelbrot law has many application in linguistics and information sciences. Some of the recent study about Zipf-Mandelbrot law can be seen in the listed references (see $[39,43]$ ). Now we state our results involving entropy introduced by Mandelbrot law by establishing the relationship with Shannon and relative entropies.

Theorem 14. Let $\mathbf{q}$ be Zipf-Mandelbrot law as defined in (72) with parameters $c \geq 0, d>0$, and $N \in\{1,2, \ldots\}$, and we have

$$
\begin{aligned}
Z_{M}(H, c, d) & =S(\mathbf{q}) \leq N \times \sum_{\ell=2}^{\mathfrak{n}-2}\left(\frac{1}{\ell !(\ell+2)(\beta-\alpha)}\right) \times \\
& \left\{\frac{(-1)^{\ell+1} \ell !}{\alpha^{(\ell+1)}} F\left(\mathbf{q},\left((\mathscr{J}+c)^{d} H_{c, d}^{N}\right),-\ln (\cdot)\right)-\frac{(-1)^{\ell+1} \ell !}{\beta^{(\ell+1)}} F\left(\mathbf{q},\left((\mathscr{J}+c)^{d} H_{c, d}^{N}\right),-\ln (\cdot)\right)\right\} .
\end{aligned}
$$

Proof. It is interesting to see that for $q_{q}$ defined in (72), $\sum_{\mathcal{f}=1}^{N} q_{\mathcal{f}}=1$. Therefore, using above $q_{\mathcal{f}}$ in Shannon entropy

(64), we get Mandelbrot entropy (70):

$$
S(\mathbf{q})=-\sum_{\mathcal{J}=1}^{N} q_{\mathcal{J}} \ln q_{\mathcal{J}}=-\sum_{\mathcal{J}=1}^{N} \frac{1}{\left((\mathscr{J}+c)^{d} H_{c, d}^{N}\right)} \ln \frac{1}{\left((\mathscr{J}+c)^{d} H_{c, d}^{N}\right)}=\frac{d}{\left(H_{c, d}^{N}\right)} \sum_{\mathcal{J}=1}^{N} \frac{\ln (\mathscr{J}+c)}{(\mathscr{J}+c)^{d}}+\ln \left(H_{c, d}^{N}\right) .
$$

Finally, substituting this $q_{\mathscr{g}}=1 /\left((\mathscr{J}+c)^{d} H_{c, d}^{N}\right)$ in Corollary 10 (ii), we get the desired result.
Corollary 12. Let $\mathbf{q}$ and $\mathbf{p}$ be Zipf-Mandelbrot law with parameters $\quad c_{1}, c_{2} \in[0, \infty), d_{1}, d_{2}>0$, and 


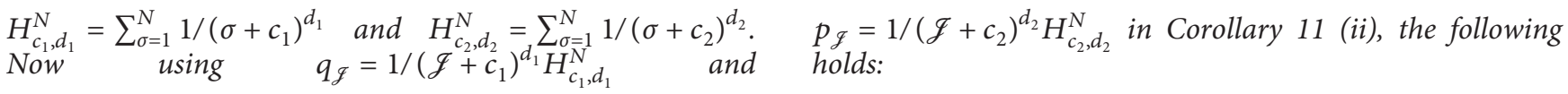

$$
\begin{aligned}
D(\mathbf{q} \| \mathbf{p}) & =\sum_{\mathcal{J}=1}^{N} \frac{1}{\left(\mathscr{J}+c_{1}\right)^{d_{1}} H_{c_{1}, d_{1}}^{N}} \ln \left(\frac{\left(\mathscr{J}+c_{2}\right)^{d_{2}} H_{c_{2}, d_{2}}^{N}}{\left(\mathscr{J}+c_{1}\right)^{d_{1}} H_{c_{1}, d_{1}}^{m}}\right) \\
& =-Z\left(H, c_{1}, d_{1}\right)+\frac{d_{2}}{H_{c_{1}, d_{1}}^{N}} \sum_{\mathcal{J}=1}^{N} \frac{\ln \left(\mathscr{J}+c_{2}\right)}{\left(\mathscr{J}+c_{1}\right)^{d_{1}}}+\ln \left(H_{c_{2}, d_{2}}^{m}\right) \\
& \leq N \sum_{\ell=2}^{\mathfrak{n}-2}\left(\frac{1}{\ell !(\ell+2)(\beta-\alpha)}\right) \times\left\{\frac{(-1)^{\ell+1} \ell !}{\alpha^{(\ell+1)}} F\left(\mathbf{q}, \frac{\left(\mathscr{J}+c_{1}\right)^{d_{1}} H_{c_{1}, d_{1}}^{N}}{\left(\mathscr{J}+c_{2}\right)^{d_{2}} H_{c_{2}, d_{2}}^{N}}, \ln (\cdot)\right)-\frac{(-1)^{\ell+1} \ell !}{\beta^{(\ell+1)}} F\left(\mathbf{q}, \frac{\left.\left.\left(\mathscr{J}+c_{1}\right)^{d_{1}} H_{c_{1}, d_{1}}^{N},-\ln (\cdot)\right)\right\} .}{\left(\mathscr{J}+c_{2}\right)^{d_{2}} H_{c_{2}, d_{2}}^{N}}\right)\right\}
\end{aligned}
$$

The Next Result for Hybrid Zipf-Mandelbrot Entropy. Further generalization of Zipf-Mandelbrot entropy is Hybrid
Zipf-Mandelbrot entropy. Let $N \in\{1,2, \ldots\}, \quad c \geq 0 \omega>0$; then, Hybrid Zipf-Mandelbrot entropy can be given as

$$
\widehat{Z}_{M}\left(H^{*}, c, d, \omega\right)=\frac{1}{H_{c, d, \omega}^{*}} \sum_{\mathcal{J}=1}^{N} \frac{\omega^{\mathscr{F}}}{(\mathscr{J}+c)^{d}} \ln \left(\frac{(\mathscr{J}+c)^{d}}{\omega^{\mathscr{F}}}\right)+\ln \left(H_{c, d, \omega}^{*}\right)
$$

where

$$
H_{c, d, \omega}^{*}=\sum_{\mathcal{J}=1}^{N} \frac{\omega^{\mathscr{g}}}{(\mathscr{J}+c)^{d}}
$$

Consider

$$
q_{\mathscr{J}}=\Psi(\mathscr{J} ; N, c, d, \omega)=\frac{\omega^{\mathscr{J}}}{(\mathscr{J}+c)^{d} H_{c, d, \omega}^{*}},
$$

which is called Hybrid Zipf-Mandelbrot law. There is a unified approach, maximization of Shannon entropy [44], that naturally follows the path of generalization from Zipf's to Hybrid Zipf's law. Extending this idea, Jakšetic et al. in [45] presented a transition from Zipf-Mandelbrot to Hybrid Zipf-Mandelbrot law by employing maximum entropy technique with one additional constraint. It is interesting that examination of its densities provides some new insights of Lerch's transcendent.

Theorem 15. Let $\mathbf{q}$ be Hybrid Zipf-Mandelbrot law as defined in (78) with parameters $c \geq 0, d, \omega>0$, and $N \in\{1,2, \ldots\}$, and we have

$$
\begin{aligned}
\widehat{Z}_{M}\left(H^{*}, c, d, \omega\right)=S(\mathbf{q}) \leq & N \sum_{\ell=2}^{\mathfrak{n}-2}\left(\frac{1}{\ell !(\ell+2)(\beta-\alpha)}\right) \times \\
& \left\{\frac{(-1)^{\ell+1} \ell !}{\alpha^{(\ell+1)}} F\left(\mathbf{q}, \frac{(\mathscr{J}+c)^{d} H_{c, d, \omega}^{*}}{\omega^{\mathcal{J}}},-\ln (\cdot)\right)-\frac{(-1)^{\ell+1} \ell !}{\beta^{(\ell+1)}} F\left(\mathbf{q}, \frac{(\mathscr{J}+c)^{d} H_{c, d, \omega}^{*}}{\omega^{\mathcal{J}}},-\ln (\cdot)\right)\right\}
\end{aligned}
$$


Proof. It is interesting to see that for $q_{g}$ defined in (78), $\sum_{\mathcal{f}=1}^{N} q_{\mathcal{g}}=1$. Therefore, using above $q_{\mathcal{g}}$ in Shannon entropy (64), we get Hybrid Zipf-Mandelbrot entropy (76):

$$
\begin{aligned}
& S(\mathbf{q})=-\sum_{\mathscr{J}=1}^{N} q_{\mathscr{J}} \ln q_{\mathscr{J}}=-\sum_{\mathscr{J}=1}^{N} \frac{\omega^{\mathscr{J}}}{(\mathscr{J}+c)^{d} H_{c, d, \omega}^{*}} \ln \frac{\omega^{\mathscr{J}}}{(\mathscr{J}+c)^{d} H_{c, d, \omega}^{*}} \\
& =\frac{-1}{H_{c, d, \omega}^{*}} \sum_{\mathcal{J}=1}^{N} \frac{\omega^{\mathscr{J}}}{(\mathcal{J}+c)^{d}}\left[\ln \left(\frac{\omega^{\mathscr{J}}}{(\mathscr{J}+c)^{d}}\right)+\ln \left(\frac{1}{H_{c, d, \omega}^{*}}\right)\right] \\
& =\frac{1}{H_{c, d, \omega}^{*}} \sum_{\mathcal{J}=1}^{N} \frac{\omega^{\mathscr{J}}}{(\mathscr{J}+c)^{d}}\left[\ln \left(\frac{(\mathscr{J}+c)^{d}}{\omega^{\mathscr{F}}}\right)+\ln \left(H_{c, d, \omega}^{*}\right)\right] \\
& =\frac{1}{H_{c, d, \omega}^{*}} \sum_{\mathcal{J}=1}^{N} \frac{\omega^{\mathscr{F}}}{(\mathscr{J}+c)^{d}} \ln \left(\frac{(\mathscr{J}+c)^{d}}{\omega^{\mathscr{F}}}\right)+\ln \left(H_{c, d, \omega}^{*}\right) .
\end{aligned}
$$

Finally, substituting this $q_{\mathscr{g}}=\omega^{\mathscr{F}} /(\mathscr{J}+c)^{d} H_{c, d, \omega}^{*}$ in Corollary 10 (ii), we get the desired result.
Corollary 13. Let $\mathbf{q}$ and $\mathbf{p}$ be Hybrid Zipf-Mandelbrot law with parameters $c_{1}, c_{2} \in[0, \infty), \omega_{1}, \omega_{2}, d_{1}, d_{2}>0$. Now using $q_{\mathscr{g}}=\omega_{1}^{\mathscr{F}} /\left(\mathscr{J}+c_{1}\right)^{d_{1}} H_{c_{1}, d_{1}, \omega_{1}}^{*}$ and $p_{\mathscr{g}}=\omega_{2}^{\mathscr{F}} /$ $\left(\mathscr{J}+c_{2}\right)^{d_{2}} H_{c_{2}, d_{2}, \omega_{2}}^{*}$ in Corollary 11 (ii), the following holds:

$$
\begin{aligned}
& D(\mathbf{q} \| \mathbf{p})=\sum_{\mathcal{J}=1}^{N} \frac{\omega_{1}^{\mathscr{J}}}{\left(\mathscr{J}+c_{1}\right)^{d_{1}} H_{c_{1}, d_{1}, \omega_{1}}^{*}} \ln \left(\frac{\omega_{1}^{\mathscr{f}}\left(\mathscr{J}+c_{2}\right)^{d_{2}} H_{c_{2}, d_{2}, \omega_{2}}^{*}}{\omega_{2}^{\mathscr{f}}\left(\mathscr{J}+c_{1}\right)^{d_{1}} H_{c_{1}, d_{1}, \omega_{1}}^{*}}\right) \\
& =-\widehat{Z}_{M}\left(H^{*}, c_{1}, d_{1}, \omega_{1}\right)+\frac{1}{H_{c_{1}, d_{1}, \omega_{1}}^{*}} \sum_{\mathcal{J}=1}^{N} \frac{\omega_{1}^{\mathscr{J}}}{\left(\mathscr{J}+c_{1}\right)^{d_{1}}} \ln \left(\frac{\left(\mathscr{J}+c_{2}\right)^{d_{2}}}{\omega_{2}^{\mathscr{g}}}\right)+\ln \left(H_{c_{2}, d_{2}, \omega_{2}}^{*}\right) \\
& \leq N \sum_{\ell=2}^{\mathfrak{n}-2}\left(\frac{1}{\ell !(\ell+2)(\beta-\alpha)}\right) \times\left\{\frac{(-1)^{\ell+1} \ell !}{\alpha^{(\ell+1)}} F\left(\mathbf{q}, \frac{\omega_{2}^{\mathscr{J}}\left(\mathscr{J}+c_{1}\right)^{d_{1}} H_{c_{1}, d_{1}, \omega_{1}}^{*}}{\omega_{1}^{\mathcal{J}}\left(\mathcal{J}+c_{2}\right)^{d_{2}} H_{c_{2}, d_{2}, \omega_{2}}^{*}},-\ln (\cdot)\right)\right. \\
& \left.-\frac{(-1)^{\ell+1} \ell !}{\beta^{(\ell+1)}} F\left(\mathbf{q}, \frac{\omega_{2}^{\mathscr{J}}\left(\mathscr{J}+c_{1}\right)^{d_{1}} H_{c_{1}, d_{1}, \omega_{1}}^{*}}{\omega_{1}^{\mathscr{J}}\left(\mathscr{J}+c_{2}\right)^{d_{2}} H_{c_{2}, d_{2}, \omega_{2}}^{*}},-\ln (\cdot)\right)\right\} .
\end{aligned}
$$

Remark 9. Similarly, we can give results for Shannon entropy, Kullback-Leibler distance, Zipf-Mandelbrot entropy, and Hybrid Zipf-Mandelbrot entropy by using generalized Giaccardi inequality defined in (54) on the same steps.

\section{Concluding Remarks}

In this paper, we gave generalization of Jensen's inequality as well as converse of Jensen's inequality by using Montgomery identity. We also formulate results for other inequalities like
Jensen-Steffensen inequality, Jensen-Boas inequality, and Jensen-Brunk inequality. We can obtain Jensen-Steffensen inequality, Jensen-Boas inequality, and Jensen-Brunk inequality by changing the assumption of Jensen's inequality. At the end, we gave applications in information theory for our obtained results, especially we gave results for Hybrid Zipf-Mandelbrot entropy for our obtained results [46].

\section{Data Availability}

No data were used to support this study. 


\section{Conflicts of Interest}

The authors declare that there are no conflicts of interest regarding the publication of this paper.

\section{Acknowledgments}

This research is supported by H.E.C. Pakistan under NRPU Project 7906 and RUDN University Strategic Academic Leadership Program.

\section{References}

[1] X. L. Zhu and G. H. Yang, "Jensen inequality approach to stability analysis of discrete-time systems with time-varying delay," in Proceedings of the American Control Conference, pp. 1644-1649, Seattle, WA, USA, June 2008.

[2] S. I. Butt, M. Klaričić Bakula, Đ. Pečarić, and J. Pečarić, "Jensen-grüss inequality and its applications for the zipfmandelbrot law," Mathematical Methods in the Applied Sciences, vol. 44, no. 2, pp. 1664-1673, 2021.

[3] M. A. Khan, D. Pečarić, and J. Pečarić, "New refinement of the Jensen inequality associated to certain functions with applications," Journal of Inequalities and Applications, vol. 76, 2020.

[4] S. Khan, M. Adil Khan, S. I. Butt, and Y.-M. Chu, "A new bound for the Jensen gap pertaining twice differentiable functions with applications," Advances in Difference Equations, vol. 1, 2020.

[5] H. Budak and M. Z. Sarikaya, "On generalized Ostrowski-type inequalities for functions whose first derivatives absolute values are convex," Turkish Journal of Mathematics, vol. 40, pp. 1193-1210, 2016.

[6] P. Cerone and S. S. Dragomir, "On some inequalities arising from Montgomery's identity," Journal of Computational Analysis and Applications, vol. 5, no. 4, pp. 341-367, 2003.

[7] S. Bernstein, "Sur les fonctions absolument monotones," Acta Mathematica, vol. 52, pp. 1-66, 1929.

[8] V. Čuljak and J. Pečarić, "Interpolation polynomials and inequalities for convex functions of higher order," Acta Mathematica, vol. 5, pp. 369-386, 2012.

[9] A. M. Fink, "Bounds of the deviation of a function from its averages," Czechoslovak Mathematical Journal, vol. 42, no. 117, pp. 289-310, 1992.

[10] J. Pečarić and V. Čuljak, "Interpolation polinomials and inequalities for convex functions of higher order," Mathematical Inequalities \& Applications, vol. 5, no. 3, pp. 369-386, 2002.

[11] J. Pečarić, V. Čuljak, and A. M. Fink, "On some inequalities for convex function of higher order," Advanced Nonlinear Studies, vol. 6, no. 2, pp. 131-140, 1999.

[12] J. Pečarić, V. Čuljak, and M. Rogina, "On some inequalities for convex function of higher order II," Advanced Nonlinear Studies, vol. 45, pp. 281-294, 2001.

[13] J. Pečarić, I. Perić, and M. Rodić-Lipanović, "Uniform treatment of Jensen type inequalities," Mathematical Reports, vol. 16, no. 66, pp. 183-205, 2014.

[14] J. Pečarić and J. Perić, "Improvement of the Giaccardi and the petrović inequality and related stolarsky type means," Annals of the University of Craiova - Mathematics and Computer Science, vol. 39, no. 1, pp. 65-75, 2012.

[15] J. Pečarić, M. Praljak, and A. Witkowski, "Linear operator inequality for $n$-convex functions at a point," Mathematical Inequalities \& Applications Appl, vol. 18, pp. 1201-1217, 2015.
[16] Z. Pavić, "Refinements of Jensen's inequality for infinite convex combinations," Turkish Journal of Inequalities, vol. 2, no. 2, pp. 44-53, 2018.

[17] R. Bibi, A. Nosheen, and J. Pečarić, "Extended Jensen's type inequalities for diamond integrals via Taylors formula," Turkish Journal of Inequalities, vol. 3, no. 1, pp. 7-18, 2019.

[18] T. Niaz, K. A. Khan, and J. Pečarić, "On refinement of Jensen's inequality for 3-convex function at a point," Turkish Journal of Inequalities, vol. 4, no. 1, pp. 70-80, 2020.

[19] A. Ekinci, A. O. Akdemir, and M. E. Ozdemir, "Integral inequalities for different kinds of convexity via classical inequalities," Turkish Journal of Science, vol. 5, no. 3, pp. 305313,2020

[20] Y. M. Chu, S. Talib, E. Set, M. U. Awan, and M. A. Noor, " $(p, q)-$ Analysis of Montgomery identity and estimates of $(p, q)$-bounds with applications," Journal of Inequalities and Applications, vol. 2021, 9 pages, 2021.

[21] M. Z. Sarikaya, H. Yaldrz, and E. Set, "On fractional inequalities via Montgomery identities," International Journal of Open Problems in Complex Analysis, vol. 6, no. 2, pp. 36-43, 2014.

[22] A. Bnouhachem, "A descent SQP alternating direction method for minimizing the sum of three convex functions," Journal of Nonlinear Functional Analysis, vol. 4, pp. 469-482, 2020.

[23] A. O. Akdemir, S. I. Butt, M. Nadeem, and M. A. Ragusa, "New general variants of Chebyshev type inequalities via generalized fractional integral operators," Mathematics, vol. 92, 2021.

[24] S. I. Butt, M. Umar, S. Rashid, A. O. Akdemir, and Y. Ming Chu, "New Hermite Jensen Mercer type inequalities via $k$-fractional integrals," Advances in Difference Equations, vol. $635,2020$.

[25] S. I. Butt, M. Nadeem, S. Qaisar, A. O. Akdemir, and T. Abdeljawad, "Hermite Jensen Mercer type inequalities for conformable integrals and related results," Advances in Difference Equations, vol. 501, 2020.

[26] A. Bnouhachem and X. Qin, "An inertial proximal PeacemanRachford splitting method with SQP regularization for convex programming," Journal of Nonlinear Functional Analysis, vol. 50, 2020.

[27] S. Hamann, "Minimality conditions for convex composite functions and efficiency conditions in vector optimization," Applied Set-Valued Analysis and Optimization, vol. 1, pp. 221-229, 2019.

[28] A. R. Khan, J. Pečarić, and M. Praljak, "Popoviciu type inequalities for n-convex functions via extension of Montgomery identityn-convex functions via extension of Montgomery identity," Analele Universitatii “Ovidius" Constanta-Seria Matematica, vol. 24, no. 3, pp. 161-188, 2016.

[29] R. P. Boas, "The Jensen-Steffensen inequality," Elektrotehn. Fak. Ser. Mat. Fiz, vol. 302-319, pp. 1-8, 1970.

[30] J. Pečarić, F. Proschan, and Y. L. Tong, Convex Functions, Partial Orderings and Statistical Applications, Academic Press, New York, NY, USA, 1992.

[31] H. D. Brunk, "On an inequality for convex functions," Proceedings of the American Mathematical Society, vol. 7, no. 5, p. $817,1956$.

[32] P. R. Beesack and J. E. Pečarić, "On Jessen's inequality for convex functions," Journal of Mathematical Analysis and Applications, vol. 110, no. 2, pp. 536-552, 1985.

[33] R. Barlow, A. Marshall, and F. Proschan, "Some inequalities for starshaped and convex functions," Pacific Journal of Mathematics, vol. 29, no. 1, pp. 19-42, 1969. 
[34] J. E. Pečarić, “On an inequality of PMV Janić," Publications de l'Institut Mathématique, vol. 28, no. 42, pp. 145-149, 1980.

[35] G. Szegö, "Über eine Verallgemeinerung des Dirichletschen Integrals," Mathematische Zeitschrift, vol. 52, no. 1, pp. 676-685, 1950.

[36] F. Giaccardi, "Su alcune disuguaglianie," Journal of Research of the National Bureau of Standards, vol. 1, no. 4, pp. 139-153, 1955.

[37] D. S. Mitrinović, J. E. Pečarić, and A. M. Fink, Classical and new inequalities in analysis. Mathematics and its Applications (East European Series), Vol. 61, Kluwer Academic Publishers Group, Dordrecht, Netherlands, 1993.

[38] I. Csiszár, "Information-type measures of diference of probability distributions and indirect observations," Studia Science Math Hungar, vol. 2, pp. 299-318, 1967.

[39] L. Horváth, D. Pečarić, and J. Pečarić, "Estimations of $\mathrm{f}$ - and rényi divergences by using a cyclic refinement of the Jensen's inequality," Bulletin of the Malaysian Mathematical Sciences Society, vol. 42, 2017.

[40] S. Kullback, Information Theory and Statistics, Wiley, New York, NY, USA, 1959.

[41] S. T. Piantadosi, "Zipf's word frequency law in natural language: a critical review and future directions," Psychonomic Bulletin and Review, vol. 21, no. 5, pp. 1112-1130, 2014.

[42] Z. K. Silagadze, "Citations and the zipf-mandelbrot law," Complex Systems, no. 11, pp. 487-499, 1997.

[43] J. Jakšetic, D. Pečarić, and J. Pečarić, "Some properties of zipfmandelbrot law and hurwitz $\zeta$-function," Mathematical Inequalities \& Applications, vol. 21, no. 2, pp. 575-584, 2018.

[44] M. Visser, "Zipf's law, power laws and maximum entropy," Mathematical Inequalities \& Applications, vol. 15, Article ID 043021, 2013.

[45] J. Jakšetic, D. Pečarić, and J. Pečarić, "Hybrid zipf-mandelbrot law," Mathematical Inequalities \& Applications, vol. 13, no. 1, pp. 275-286, 2019.

[46] D. V. Widder, "Completely convex functions and Lidstone series," Transactions of the American Mathematical Society, vol. 51, p. $387,1942$. 